\title{
Número de racimos y la sostenibilidad económica del tomate bajo condiciones semicontroladas
}

\section{Bunches number and economic sustainability of tomato under semi- controlled conditions}

\author{
Olver Suárez ${ }^{1}$, Alejandro Hurtado-Salazar²*, Nelson Ceballos-Aguirre² \\ Recibido para publicación: Agosto 1 de 2017 - Aceptado para publicación: Noviembre 23 de 2017
}

\begin{abstract}
RESUMEN
Estudios relacionados con la productividad del tomate respecto a la relación entre la eliminación de racimos y su sostenibilidad económica son escasos. El presente estudio se realizó con el fin de evaluar el efecto del número de racimos sobre el rendimiento, calidad y rentabilidad del cultivo de tomate hibrido Torrano ${ }^{\circledR}$ bajo condiciones semicontroladas en dos invernaderos de $12 \mathrm{~m}$ x 40m $\left(480 \mathrm{~m}^{2}\right)$. Éste se llevó a cabo en la granja Tesorito, propiedad de la Universidad de Caldas, Manizales bajo un diseño de bloques completos al azar con cuatro despuntes: 6; 8 y 10 racimos/planta y sin despunte con crecimiento indeterminado, cada tratamiento contó con seis repeticiones y la unidad experimental fue de cinco plantas. Se evaluó la calidad tamaño del fruto y el rendimiento por planta y por hectárea. Las plantas sin poda con crecimiento indeterminado mostraron los valores más altos en las diferentes calidades de la fruta como primera, segunda, tercera y el rendimiento total con valores de 2236, 1666, 750 y 6883 g/planta respectivamente. Igualmente genero los ingresos netos más altos con USD \$ $27.241 \mathrm{ha} / \mathrm{ciclo}$, sin embargo en términos porcentuales la calidad aumenta a medida que se disminuye el número de racimos por planta. Por tanto, se concluye que no se debe realizar podas de racimos en tomate hibrido Torrano $\mathbb{R}$ bajo condiciones semicontroladas en la zona de estudio.
\end{abstract}

Palabras clave: Pruning, quality of the fruit, cost of production, pruning.

\begin{abstract}
Studies related to tomato productivity regarding the relationship between the elimination of flower clusters and their economic sustainability are scarce. The present study was conducted in order to evaluate the effect of the number of bunches on the yield, quality and profitability

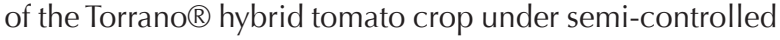
conditions in greenhouses of $12 \mathrm{~m} \times 40 \mathrm{~m}(480 \mathrm{~m} 2)$ for a total area of $960 \mathrm{~m}^{2}$. This was carried out in the Tesorito farm, property of the University of Caldas, Manizales under a design of complete blocks at random with four cut: 6; 8 and 10 bunches / plant and without cut with indeterminate growth, each treatment had six repetitions and the experimental unit was five plants. The quality of the fruit and the yield per plant and per hectare were evaluated. The plants without pruning with indeterminate growth showed the highest values in the different qualities of the fruit like first, second, third and the total yield with values of 2236, 1666, 750 and $6883 \mathrm{~g}$ / plant respectively. Equally, genre higher net income with \$27,241 has / cycle, however in percentage terms quality increases as the number of bunches per plant is reduced. Therefore, it is concluded that clusters should not be pruned in Torrano ${ }^{\circledR}$ hybrid tomato under semicontrolled conditions in the study area.
\end{abstract}

Key words: Pruning, quality of the fruit, cost of production, pruning.

\footnotetext{
${ }^{1}$ Administrador de Empresas Agropecuarias. Universidad de Caldas, Facultad de Ciencias Agropecuarias, Manizales - Caldas, Colombia.

$2^{*}$ Ph.D, Universidad de Caldas, Facultad de Ciencias Agropecuarias, Departamento de Producción Agropecuaria, Universidad de Caldas, Calle 65 № 26 -10, Apartado aéreo 275, Manizales - Caldas, Colombia. Correo alhuza@gmail.com
} 


\section{INTRODUCCIÓN}

La importancia de las hortalizas en la dieta humana se debe al hecho de que no sólo son fuente de carbohidratos y proteínas, sino también un excelente suministro de vitaminas y minerales. El tomate (Solanum lycopersicum L.) es la principal hortaliza en volumen consumida in natura en el mundo, siendo una de las principales fuentes de licopeno, un importante compuesto antioxidante y anticancerígeno, además de fuente de ácidos (ácido acético, ácido láctico y ácido málico, Vitamina C y de rasgos de potasio, hierro y fósforo (Hachmann et al., 2014; Monteiro et al., 2008).

En el segmento de tomate de mesa, la calidad del fruto y la oferta en la temporada baja son factores que pueden garantizar el éxito de la producción (Guimarães et al., 2007). Una de las formas de mejorar la calidad y apariencia del tomate es la adopción de técnicas adecuadas de manejo del cultivo (Herrera et al., 2015).

El cultivo en ambiente protegido proporciona un aumento en los ingresos, así como la obtención de productos de mejor calidad (Arbelaez et al., 2016). A través de este tipo de cultivo es posible reducir la estacionalidad de producción, además de posibilitar el control parcial de factores responsables por el crecimiento y desarrollo de las plantas (Hachmann et al., 2014).

El cultivo dentro del ambiente debe aprovechar al máximo el área disponible, debido a los altos costos de instalación de las estructuras y al alto nivel tecnológico aplicado. Una forma de aumentar el aprovechamiento del área de cultivo es aumentar la densidad de plantación y las podas. Esta última practica cultural, podría auxiliar la rentabilidad del cultivo, cambiando la participación de las calidades en la cosecha, y la disminución en la duración del cultivo para aumentar la rotación del mismo (Herrera et al., 2015). Según Hoyos y HurtadoSalazar (2017), cuando se realizan podas en las plantas de forma general, y como es descrito en otras especies frutales (Mendonça y Mendeiros, 2011), es necesario el desbrote, para concentrar toda la energía en la floración, fructificación y mejorar la calidad del fruto. Para el cultivo de tomate el despunte es una práctica en la que se quita la yema apical de los renuevos. Esto limita el número de racimos por renuevos y permite el aumento del tamaño de los frutos remanentes(Machado et al., 2007), principalmenteen losracimossituadoseneltercio superior de las plantas (Wamser et al., 2007).

Estudios de la productividad del tomate respecto a la relación entre la eliminación de racimos y su sostenibilidad económica son escasos. Por lo tanto, este trabajo tuvo como objetivo evaluar el efecto del número de racimos sobre la producción por planta y la sostenibilidad económica en el cultivo del tomate bajo condiciones semicontroladas en invernadero bajo control de los factores como riego y nutrición de las plantas..

\section{MATERIALES Y MÉTODOS}

El estudio se realizó en la Granja Tesorito de la Universidad de Caldas, localizada en el municipio de Manizales, departamento de Caldas (Colombia); con altitud de 2.340 msnm, temperatura media anual de $17,5{ }^{\circ} \mathrm{C}$, humedad relativa del $78 \%$, precipitación anual de 2.000 $\mathrm{mm}$, brillo solar de 1.473 horas año-1 (Anuario Meteorológico Cafetero, 2012 y 2013) y suelos Andisoles derivados de cenizas volcánicas con textura francoarenosa ricos en materia orgánica.

Se empleó el tomate híbrido Torrano®. Se sembraronlassemillasenbandejasde128lóculos. El sustrato empleado fue turba Klasman $\mathrm{N}^{\circ} 3$.

Dos días antes del trasplante las plántulas fueron sometidas a un proceso de endurecimiento del sistema radicular mediante estrés hídrico; una vez establecido en el invernadero el suelo se regó a capacidad de campo. 
El trasplante se realizó 30 días después de realizado el semillero en dos módulos (invernaderos) de $12 \mathrm{~m} \times 40 \mathrm{~m}\left(480 \mathrm{~m}^{2}\right)$ para un área total de $960 \mathrm{~m} 2$ con un "diseño en capilla a dos aguas" con una estructura hecha en guadua y una cubierta en plástico Agroclear. Se utilizó acolchado plástico negronegro como cobertura. Las distancias de siembra utilizadas fueron 1,2 $\mathrm{m}$ entre surcos y $0,4 \mathrm{~m}$ entre plantas para una densidad de población de 20.833 plantas por hectárea.

La fertilización fue convencional (edáfica) cada 20 días, adicionalmente, se realizó la aplicación de agua por el sistema de riego de acuerdo con lo reportado por Arbeláez et al. (2016). El tutorado de las plantas se realizó de sujetando un hilo de polipropileno (Ref. 1500 X 6000), a un extremo de la zona basal de la planta y la otra punta sujetada a un alambre situado a una altura de 2,2 m desde el suelo, esta labor (tutorado) se hizo en la semana número tres después del trasplante. A medida que la planta fue creciendo se guío el hilo tutor (semanalmente).

El diseño experimental fue bloques completos al azar con cuatro tratamientos: $6,8,10$ racimos y crecimiento indeterminado el cual se tuvo a libre crecimiento hasta la altura del alambre $(2,2$ $\mathrm{m})$, cada tratamiento contó con seis repeticiones y la unidad experimental fue de cinco plantas.

Respecto a las labores culturales, la primera poda se efectuó a los 18 días después del trasplante, en adelante se realizó semanalmente. El deshoje bajero se realizó al iniciar el Ilenado del primer racimo de frutos, semanalmente se eliminaron las hojas bajera, hasta dejar dos hojas por debajo del primer racimo de frutos. El descope o corte de la yema apical de las plantas, se realizó cuando ésta llegaba al número de racimos correspondientes a cada tratamiento $(6,8,10$ racimos y crecimiento indeterminado). El inicio de cosecha se realizó a los 115 días después del trasplante; el fruto se desprendió del pedúnculo dejando el cáliz. El índice de cosecha utilizado para esta labor se definió por el grado de madurez del fruto, siendo éste del 25\% del cambio de color de verde a verde amarillento, la recolección se hizo una vez por semana y esta labor se realizó durante el número de días de acuerdo con el tratamiento correspondiente.

Las variables evaluadas fueron: rendimiento por calidad (g/planta) (extra, primera, segunda, tercera y pérdidas), costos totales por tratamiento, producción por planta (g/planta) y rendimiento $\left(\mathrm{kg} \mathrm{ha}^{-1}\right)$. Finalmente se realizó el análisis de rentabilidad de cada tratamiento para evaluar su sostenibilidad económica. El análisis de la información se realizó a través de análisis de varianza y prueba de promedios tipo Duncan mediante el procedimiento GLM de SAS (SAS Institute Cary N.C; versión 9,0).

\section{RESULTADOS Y DISCUSIÓN}

En términos de cantidad por planta, se observó para la variable calidad "extra" un mejor comportamiento para el tratamiento sin poda de racimos (crecimiento indeterminado), seguido del tratamiento con 10 racimos por planta, con valores de 1921 y 2202 g/planta respectivamente (Tabla 1). En contraste los tratamientos con 6 racimos y 8 racimos por planta arrojaron los valores de producción de calidad "extra" más bajos y a su vez por debajo del promedio (1848 g/planta). Sin embargo en términos porcentuales, los porcentajes más altos de calidad extra se presentaron en los tratamientos con poda de racimos, alcanzando un $37 \%$ de calidad extra en los mismos, frente a un $27 \%$ en el tratamiento sin poda de racimo (crecimiento indeterminado).

Comportamiento similar obtuvo el tratamiento con crecimiento indeterminado para las variables de calidad (primera, segunda, tercera y producción total) con valores de 2236, 1666, 750 y 6883 g/planta respectivamente, con diferencias significativas respecto a los demás tratamientos $(p<0,05)$. De igual forma, presentó 
Tabla 1. Pruebas comparativas de promedios Duncan sobre las variables de calidad, producción (g/planta) y rendimiento ( $\mathrm{kg}$ ha-1) en el cultivo de tomate (Solanum lycopersicum L.) bajo condiciones semicontroladas.

\begin{tabular}{|c|c|c|c|c|c|c|c|c|}
\hline \multirow{2}{*}{$\begin{array}{l}\text { Numero de racimos } \\
\text { por planta }\end{array}$} & \multicolumn{5}{|c|}{ Calidad (g/planta) } & \multicolumn{2}{|c|}{ Producción (g/planta) } & \multirow{2}{*}{$\begin{array}{l}\text { Rendimiento } \\
\text { (kg ha-1) }\end{array}$} \\
\hline & Extra & Primera & Segunda & Tercera & Pérdida & Neta & Total & \\
\hline Indeterminado & 1921 a & 2236 a & 1666 a & $750 \mathrm{a}$ & $750 \mathrm{a}$ & 6574 a & $6883 \mathrm{a}$ & $143400 \mathrm{a}$ \\
\hline 10 & $2202 \mathrm{a}$ & $1815 b$ & $1134 b$ & $551 \mathrm{~b}$ & $551 \mathrm{~b}$ & $5702 \mathrm{~b}$ & 5994 b & $124900 \mathrm{~b}$ \\
\hline 8 & $1674 \mathrm{~b}$ & $1361 \mathrm{c}$ & $1023 \mathrm{~b}$ & $519 \mathrm{~b}$ & $519 \mathrm{~b}$ & $4578 \mathrm{c}$ & $4864 \mathrm{c}$ & $101300 \mathrm{c}$ \\
\hline 6 & $1597 \mathrm{~b}$ & 1193 c & 823 c & $358 \mathrm{c}$ & $358 \mathrm{c}$ & $3972 d$ & $4228 d$ & $88100 d$ \\
\hline Promedio & 1848 & 1651 & 1161 & 544 & 544 & 5206 & 5492 & 114400 \\
\hline$\sigma$ & 236,61 & 407,12 & 311,86 & 139,37 & 139,37 & 1004,31 & 1022,12 & 21298,75 \\
\hline
\end{tabular}

Las medias seguidas por la misma letra en la columna no difieren por la prueba de Tukey al $5 \%$ de probabilidad. $\sigma=$ desviación estándar.

el valor más alto en cantidad de pérdidas con 309 g/planta (Tabla 1).

Wamser et al. (2007), encontraron que sólo hay diferencia en la productividad total, calidad extra y descarte en dos años consecutivos de evaluación (2006/07) y la productividad de los frutos comerciales no difirió entre las alturas de despunte de racimos $(5,7,9$ y sin despunte). Estos resultados son diferentes de los obtenidos por Seleguini et al. (2006) y Machado eet al. (2007), que observaron reducción de la productividad de frutos con la disminución del número de racimos por planta. Sin embargo, el número de racimos por planta probados por estos autores fueron menores que los del presente experimento.

La posibilidad de despuntar por encima del $5^{\circ}$ racimo de cada vástago, sin haber pérdidas en la productividad comercial de frutos, trae una serie de beneficios para el cultivo y para el productor. Según Seleguini et al. (2006), la disminución del número de racimos por planta reduce el ciclo de cultivo y la altura de las plantas, proporcionando menor utilización de mano de obra durante el ciclo de cultivo y el menor uso de los defensivos agrícolas. Además, la menor altura de las plantas permite una mayor eficiencia en el control de plagas y enfermedades y mejor distribución de luz en el cultivo (Seleguini et al., 2006). La reducción del ciclo del cultivo, a través del despunte, puede ser utilizada como un mecanismo de escape contra la incidencia de enfermedades (Streck et all., 1998).
La producción total para el tratamiento crecimiento indeterminado, fue superior a los reportados por Arbelaez et al. (2016), los cuales en sus mejores tratamientos reportaron valores de 6010 y 4783 g/planta, con este mismo hibrido y en las mismas condiciones agroecológicas.

El mejor rendimiento se presentó con el tratamiento de crecimiento indeterminado con $143400 \mathrm{~kg} \mathrm{ha}^{-1}$, seguido por el tratamiento con 10 racimos con $124900 \mathrm{~kg} \mathrm{ha}^{-1}$, este superado por el indeterminado en $18500 \mathrm{~kg}$ hga-1, lo cual en términos económicos mejora los ingresos netos e incrementa la relación beneficio costo (Tabla 1). Estos resultados difieren de los observados por Machado et al. (2007), en que la reducción del número de racimos por planta aumentó la masa media de frutos. Sin embargo, para las mayores alturas de desprendimiento evaluadas en el presente experimento, muchos de los frutos localizados en los racimos superiores no alcanzaron el punto de cosecha al final de la cosecha. Estos frutos fueron eliminados con la parte vegetativa, según lo observado por Streck et al. (1998) y, por poseer menor tamaño (Carvalho y Tessarioli, 2005), acaban no contribuyendo con la disminución de la masa media de frutos en los tratamientos con mayor altura de despuntar o sin la realización del despunte.

De acuerdo con Bogiani et al. (2008), la utilización de poda apical después de la segunda inflorescencia, asociada a la densidad de 50.000 plantas, posibilita la concentración de la cosecha en un período de siete semanas y mayor productividad, debido al menor aborto de 
flores, en función de la coincidencia del período productivo con época climáticamente más adecuada. No hubo efecto del sistema de cultivo sobre la masa media de materia fresca y en la distribución de los frutos por clases de tamaño.

De acuerdo con Arbelaez et al. (2016), el rubro con mayor participación en los costos de producción del cultivo de tomate, son los insumos generales como semillas, plástico mulch, agroquímicos, fertilizantes y enmiendas lo cual oscilan entre $39,91 \%$ y $42,49 \%$ del total de los costos de producción. Los costos de amortización por ciclo de 8 meses de invernadero varían entre $8,01 \%$ y $8,12 \%$ del total de los costos, ocupando así el cuarto lugar de mayor peso en los costos.

En tanto, los mayores costos de producción en estos materiales se debe a la exigencia alta de mano de obra para las labores de tutorado y cosecha principalmente (Sánchez-Del Castillo et al., 2014).

En un estudio realizado por Perilla et al. (2011), con el objetivo de caracterizar las condiciones sociales, técnicas y económicas del sistema de producción de tomate bajo invernadero en los municipios de Guateque, Sutatenza y Tenza del departamento de Boyacá (Colombia) destacan que el costo total de mano de obra, por concepto de mantenimiento del cultivo, alcanza los USD\$13.821 por hectárea (ha), para nuestro estudio el mantenimiento alcanzo valores entre USD\$23.793 y 29.310 por ha.

Estos mismos autores resaltan a la cosecha como la operación del proceso de producción que implica mayor costo, ya que esta se realiza dos veces por semana y en ella se utiliza una cantidad considerable de jornales similar a la desarrollada en este estudio, ascendiendo a USD\$11.182 por ha. Igualmente le sigue en costo la poda de mantenimiento, la que se realiza semanalmente y se utiliza en promedio de tres a cuatro jornales. Resultados similares obtuvieron
Testa et al. (2014), evaluando la producción de tomate en 30 invernaderos en Italia.

Los costos de producción por hectárea más bajos fueron los alcanzados con el tratamiento 6 racimos con USD\$23793 ha/ciclo, a su vez fue el tratamiento menos productivo ya que generó un ingreso neto de USD\$ 10344 ha/ ciclo, en contraste, el tratamiento crecimiento indeterminado, presentó los costos más altos de producción con USD\$29310 ha/ ciclo, sin embargo por ser el tratamiento más productivo, generó los ingresos netos más altos de USD\$ $27241 \mathrm{ha} /$ ciclo; esto indica una diferencia de USD\$ 16896 ha/ciclo entre ambos tratamientos, representando un 163\% más rentabilidad que el tratamiento con 6 racimos. Para los tratamientos con 10 y 8 racimos presentaron diferencias de USD \$ 4827 y USD\$13103, respectivamente, respecto el de crecimiento indeterminado (Figura 1).

El tratamiento sin poda - crecimiento indeterminado fue el que mejor productividad presentó cuyo margen unitario de producción fue de USD\$ 0,21 por $\mathrm{Kg}$, a diferencia del tratamiento con 6 racimos/planta (tratamiento de menor productividad) cuyo margen unitario de producción fue USD\$ 0,29 por $\mathrm{Kg}$, indicando que al momento de ofertar el producto, reduce las posibilidades o capacidad competitivas dentro del mercado destino.

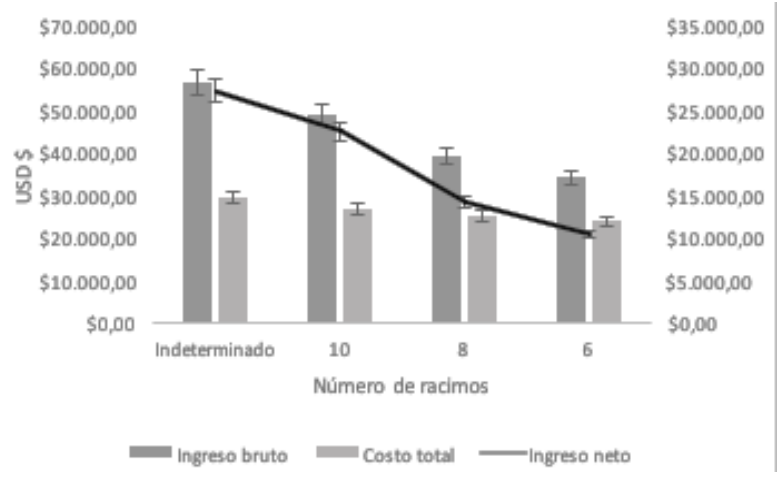

Figura 1. Efecto del número de racimos sobre la rentabilidad del cultivo de tomate (Solanum lycopersicum L.) bajo condiciones semicontroladas. 


\section{CONCLUSIONES}

Las plantas de crecimiento indeterminado (sin poda de racimos) presentaron los valores más altos en cantidad, sin embargo en términos porcentuales la calidad aumenta a medida que se disminuye el número de racimos por planta.

Los costos de producción por hectárea más bajos se alcanzaron en el tratamiento con 6 racimos planta-1 (USD\$23.793 ha/ciclo) siendo a su vez el tratamiento menos productivo con un ingreso neto de USD\$ $10.344 \mathrm{ha} /$ ciclo, en contraste, el tratamiento crecimiento indeterminado, que presentó los costos más altos de producción (USD\$ 29.310 ha/ciclo), a su vez, generó los ingresos netos más altos de USD \$ $27.241 \mathrm{ha} /$ ciclo; indicando que, con una diferencia USD\$ 16.896 entre ambos tratamientos; es el tratamiento más sostenible económicamente, para condiciones agroecológicas como las de la zona de estudio.

\section{REFERENCIAS}

Anuario meteorológico Cafetero. 2012 y 2013. Federación Nacional de Cafeteros de Colombia. Cenicafé, Chinchiná (Colombia).

Arbelaez, L., Rivera, J., Hurtado-Salazar, A. y Ceballos Aguirre, N. 2016. Technical and Economic Evaluation of Three Types of Tomato Nutrient Solutions under Semi-Controlled Conditions. Journal of Agricultural Science 8(8): 68-78.

Bogiani, J., Anton, C., Seleguini, A., Faria, M. y Seno, S. 2008. Poda apical, densidade de plantas e cobertura plástica do solo na produtividade do tomateiro em cultivo protegido. Bragantia, 67(1): 145-151.

Carvalho, L. y Tessarioli, J. 2005. Produtividade de tomate em ambiente protegido, em função do espaçamento e número de ramos por planta. Horticultura Brasileira 23: 986-989.
Guimarães, M., Silva, D., Fontes, P., Caliman, F., Loos, R. y Stringheta, P. 2007. Produção e sabor dos frutos de tomateiro submetidos a poda apical e de cachos florais. Horticultura Brasileira 25: 265269.

Hachmann, T., Echer, M., Dalastra, G., Vasconcelos, E. y Guimarães, V. 2014. Cultivo do tomateiro sob diferentes espaçamentos entre plantas e diferentes níveis de desfolha das folhas basais. Bragantia 73(4): 399-406.

Herrera, H., Hurtado-Salazar, A. y CeballosAguirre, N. 2015. Estudio técnico y económico del tomate tipo cereza élite (Solanum lycopersicum L. var. Cerasiforme) bajo condiciones semicontroladas. Revista Colombiana de Ciencias Hortícolas 9(2): 290-300

Hoyos, J. y Hurtado-Salazar, A. 2017. Poda temprana de brotes laterales en el inicio de la floración de papaya Tainung 1. Temas Agrarios 22(2): 52-58.

Machado, A., Alvarenga M. y Florentino, C. 2007. Produção de tomate italiano (saladete) sob diferentes densidades de plantio e sistemas de poda visando ao consumo in natura. Horticultura Brasileira 25: 149-153.

Mendonça, V. y Mendeiros, L. 2011. Importância da fruticultura, poda das árvores frutíferas, propagação das plantas frutíferas. Boletim I. Mossoró, R

Monteiro, C., Balbi, M., Miguel, O., Penteado, P. y Haracemiv, S. 2008. Qualidade nutricional e antioxidante do tomate "tipo italiano". Revista Alimentos e Nutrição 19: 25-31.

Perilla, A. Rodríguez, L. y Bermúdez, L. 2011. Estudio técnico-económico del sistema de producción de tomate bajo invernadero en Guateque, Sutatenza y Tenza (Boyacá). Revista colombiana de ciencias hortícolas, 5(2): 220-232.

Sánchez-Del Castillo, F., Moreno-Pérez, E., Pineda-Pineda, J., Osuna, J., RodríguezPérez, J. y Osuna-Encino, T. 2014. Producción hidropónica de jitomate (Solanum lycopersicum L.) con y sin recirculación de la solución nutritiva. Agrociencia 48: 185-197. 
Seleguini, A., Seno, S. y Faria M. 2006. Espaçamento entre plantas e número de racimos para tomateiro em ambiente protegido. Acta Scientiarum. Agronomy 28(3): 359-363.

Streck, N., Buriol, G., Andriolo, J. y Sandri, M. 1998. Influência da densidade de plantas e poda apical drástica na produtividade do tomateiro em estufa de plástico. Pesquisa Agropecuária Brasileira 33: 1105-1112.
Testa, R., Trapani, A., Sgroi, F. y Tudisca, S. 2014. Economic Sustainability of Italian Greenhouse Cherry Tomato. Sustainability, 6, 7967-7981.

Wamser A., Mueller S., Becker, W. y Santos, J. 2007. Produção do tomateiro em função dos sistemas de condução de plantas. Horticultura Brasileira 25: 238-243. 